\title{
The importance of surface finish to energy performance
}

\author{
Geoff B. Smith*, Angus R. Gentle, Matthew D. Arnold, Marc A. Gali, and Michael B. Cortie \\ School of Mathematical and Physical Sciences, University of Technology, Sydney, PO Box 123, Broadway, NSW 2007, Australia
}

Received: 11 January 2017 / Received in final form: 5 July 2017 / Accepted: 28 August 2017

\begin{abstract}
Power generation in solar energy systems, thermal control in buildings and mitigation of the Urban Heat Island problem, are all sensitive to directional response to incoming radiation. The radiation absorption and emission profile also plays a crucial role in each system's response and depends strongly on surface finish. This important sensitivity needs wider recognition in materials data sheets, system modeling, plus in materials and environmental engineering. The impact of surface roughness on thermal response of natural and man-made external environments is examined. Important examples will be given of the role of surface finish within each class. Total emittance links to the way surface finish influences directional emittance $E(\theta)$. Smooth surface thermal emittance on PV module covers, many solar absorbers, some roof paints, polished concrete, and glass windows can be up to $15 \%$ different from insulator results based on fully diffuse models of the same material. Widespread evidence indicates smooth metals and low- $E$ solar absorber surfaces cool faster, and smooth insulators slower than previously thought. Matt paint is cooler than low sheen paint under the same solar heating impacts and normal concrete cooler than polished. Emittance for water is the prime environmental example of oblique impacts as it reflects strongly at oblique incidence, which leads to a significant drop in $E(\theta)$. Ripples or waves however raise water's average emittance. A surprise in this work was the high sensitivity of total $E$ and its angular components to roughness in the depth range of $0.1-0.8 \mu \mathrm{m}$, which are well under ambient thermal IR wavelengths of 3-30 $\mu \mathrm{m}$ but common in metal finishing. Parallel energy flows such as evaporation and convective cooling vary if emittance varies. Thermal image analysis can provide insights into angular radiative effects.
\end{abstract}

\section{Introduction}

Urban surfaces, both natural and constructed, play an increasingly important role in the thermal comfort and health of city occupants since, along with the materials used and local climate, surfaces dictate the overall thermal balance and precinct temperatures. That is, they determine how much solar energy and incoming atmospheric radiation is absorbed and converted to heat, how much is reflected straight back into space, and how much heat is thermally emitted. One of the most common approximations in urban thermal studies and also in roof and wall building simulations is to assume that surfaces have emittance $E$ around 0.90 . In reality we will show a rich variety with $E$ ranging typically from around $0.70-0.95$ with much of this range down to surface finish. Since glass facades are now a dominant part of city building facades, an accurate value of their thermal emittance is especially important. Our recent work on solar cell covers [1] indicates that smooth glass emittance is significantly lower than commonly thought. There are also some important low- $E$

\footnotetext{
* e-mail: g.smith@uts.edu.au
}

exceptions when bare or thinly coated metal is used. Low emittance surfaces have important roles to play in solar thermal technology and in buildings for solar control and insulating glazing. As opposed to bare glass, we will show that reported $E$ values for many low $E$ surfaces are below their true values. Smooth conducting material emittance is higher than Lambertian-based optical models since conducting materials behave differently to dielectrics in the oblique regime: their reflectance falls at oblique angles whereas that of insulators rises.

The thermal changes linked to these optical responses also influence heat gain and cooling by convection, condensation and evaporation, so a full study is needed before conclusions on temperature can be reached. The thermal impact of surface texture variations of natural surfaces is important and dynamic, covering thermal flows involving vegetation, clay, soil, sand, rivers, lakes, harbors and estuaries. The analysis that follows is applicable to both construction materials and many natural surfaces. The extensive list of water bodies is included because the surface texture of water varies from smooth to very rough depending on wind conditions. It is not often realized that the thermal emittance properties of water are strongly wind dependent. Not only does forced convection induce 

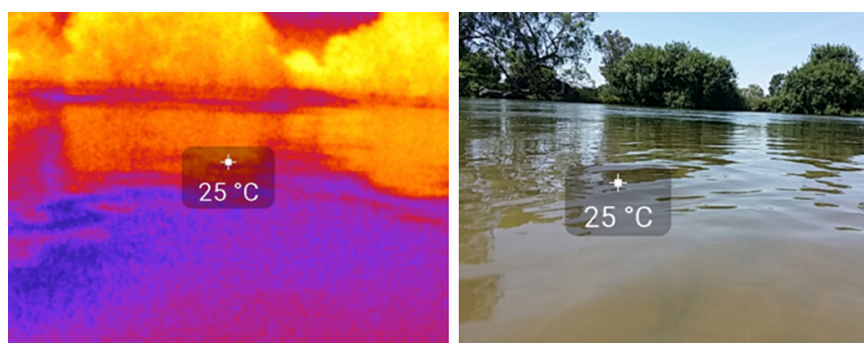

Fig. 1. A thermal image (left) and visible image (right) showing the ability of nearly smooth river water to reflect incident thermal radiation produced from warmer trees on the river bank to produce well defined thermal mirror images.

more evaporation but rates of thermal emission rise also. The angle of incidence dependence of both solar and infrared absorptance is very important. Smooth surfaces such as glass and still water are commonly assumed to absorb and emit IR strongly but actually they reflect it strongly at oblique incidence as seen in Figure 1 for water. Mirror-like impacts on thermal radiation for still water mean reduced emittance and less radiative cooling. As a consequence greater rates of evaporation occur. Figure 1 also demonstrates that thermal imaging surveys can easily be misleading when surfaces reflect radiant heat incident from nearby sources.

We will consider the role of surface finish in countering the Urban Heat Island problem and basics on the 3dimensional link between the angular dependence of absorptance and thermal flows in terms of two models that describe different spatial aspects and impacts of heat flow. Following sections will look at examples for various finishes on metals with specific results on glass and water. We conclude the introduction with a list of materials whose emittance can vary according to the different surface manifestations in the list. Smooth composites may also vary due to sub-surface scattering and absorption:

- glazing: low- $E$, standard, polymers, clear and diffuse;

- concrete: normal and polished;

- paints: matt, low sheen, high sheen, glazed;

- water: still, rippled, wind blown and rough;

- snow and ice: powder and dry, smooth, rough;

- metal: various production and finishing techniques;

- natural surfaces: sand, clay, gravel, soil, grass, crops, animal fur and skin.

\section{2. Methodology - surfaces to counter the Urban Heat Island}

City locations can be up to $3-10{ }^{\circ} \mathrm{C}$ warmer than rural and natural areas in the same climate zones, with wide ranging negative consequences [2]. Cool and super-cool surfaces have the ability to reduce local outdoor air temperatures and as a result also reduce energy consumption used for cooling $[3,4]$. The best cool paints are highly diffused at visible and solar radiations. They reflect solar and absorb thermal IR strongly as required at most angles of incidence. Such paint is illustrated in Figure 2 which shows a roof that is half-coated with such a paint showing its "cool" performance at all exit angles in contrast with a galvanized, partly oxidized un-coated section, which emits less energy obliquely and is much hotter.

Our custom-coated surface, whose near ideal solar and emittance properties extended to high angles, was the first open surface to stay sub-ambient under the summer sun [5]. Its reflectance spectrum and image is in Figure 3. Reflectance is plotted relative to the solar spectrum, black body spectrum and clear sky window (hence atmospheric) transmittance zone. The material's most important feature for daytime cooling is its albedo of $97 \%$.

This custom surface's unusual response to high angles (relative to smooth surfaces in general) is due to its complex multilayered nano-substructure [5]. Thus "smooth surface finish" from a thermal response perspective means not only having a smooth interface but also uniform composition to at least a skin depth across both solar and Planck IR spectra.

\section{Results - surface finish and emittance}

The spread of emitted radiation is described in two ways: as free flow, or as the amount incident on a remote receiver (called projected flow). Radiative cooling involves both free outflow and projection of inflow from other emitters. We have recently developed a novel planar (non-cavity) approach to modeling both flows into all solid angle zones. This detailed analysis [1,6], along with application of thermodynamic restraints, points clearly to the need to use free flow to define the heat loss component. Two hemispherical emittances $E_{e}(2 \pi)$ and $E_{p}(2 \pi)$ and two partial hemispherical emittances $E_{e}(\Delta \Omega)$ and $E_{p}(\Delta \Omega)$ emerge. $\Delta \Omega$ for this purpose is the solid angle subtended by the hemispherical cap which extends from the normal $\left(\theta=0^{\circ}\right)$ to exit angle $\theta$ degrees to the normal and covering the full axial span $\Delta \varphi=2 \pi$. Thus for use in defining partial hemispherical emittance we use $\Delta \Omega=2 \pi(1-\cos \theta)$. Heat outflow is given by equation (1) at $T(\mathrm{~K})$ with $\sigma$ the StefanBoltzmann constant and $E_{e}$ the free flow hemispherical emittance into solid angle $2 \pi$ steradians as defined by equation (2). Replacing $P_{p}$ and $E_{p}(2 \pi)$ for $P_{e}$ and $E_{e}(2 \pi)$ in equation (1) also requires the factor $0.5 \sigma T^{4}$ while $E_{p}(2 \pi)$ requires adding " $\cos \theta$ " into equation (2). The model in use thus required a factor 2.0 multiplying $P_{p}$ which was added to normalize total output to total free output for Lambertian emitters. Basic projected flow does not have this factor of 2.0, whose addition violates the second law of thermodynamics and some optical observations when spatial spread is broken down into angular components [6].

$$
\begin{gathered}
P_{e}(2 \pi, T)=E_{e}(2 \pi, T) \sigma T^{4} \quad \mathrm{~W} \mathrm{~m}^{-2} \\
E_{e}(2 \pi, T)=\frac{1}{2 \pi} \int_{0}^{2 \pi} d \varphi \int_{\theta}^{\pi / 2} d \theta E(\theta, T) \sin \theta .
\end{gathered}
$$

Using free flow one finds oblique angles of incidence (or exit) have a much greater impact than in projection models. As a result surface finish has greater impact on 


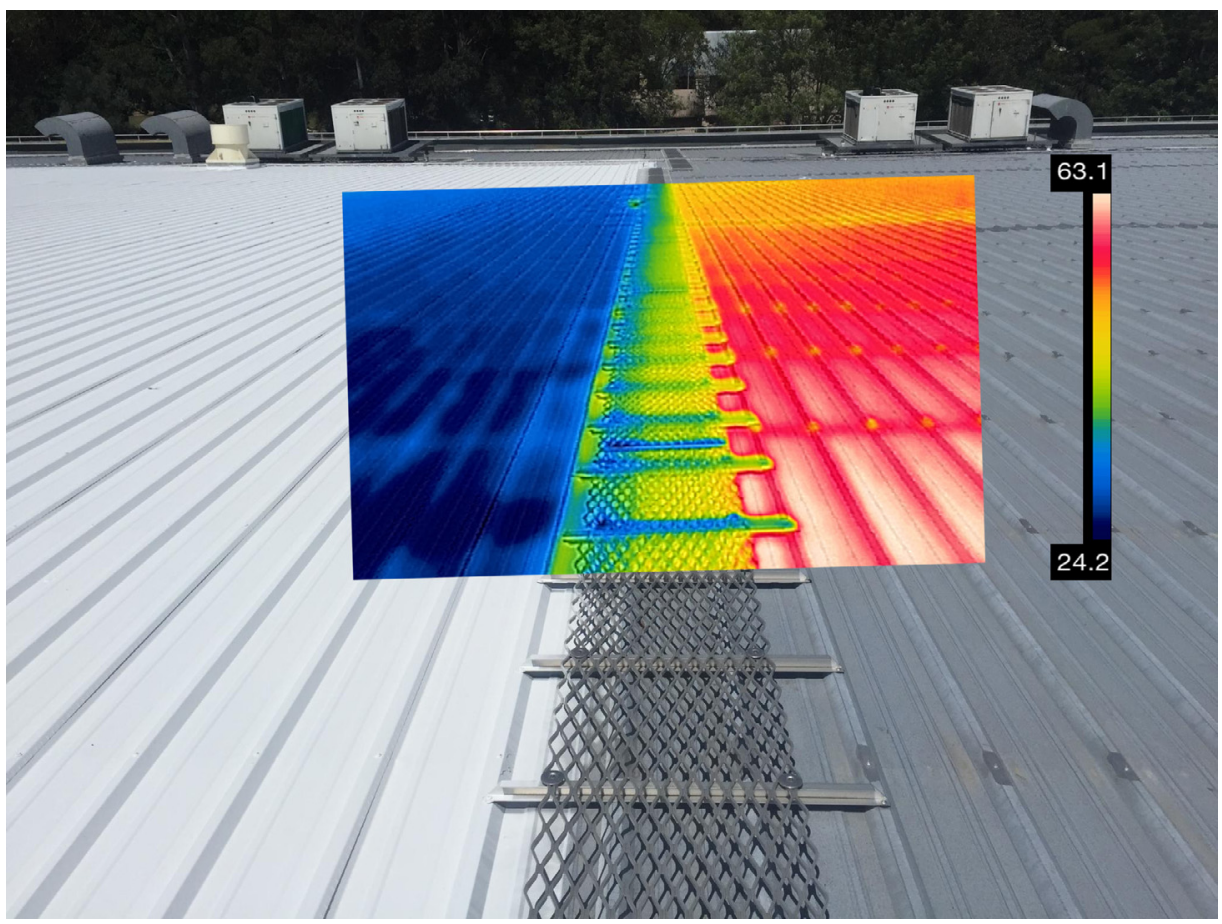

Fig. 2. A cool, diffuse, high albedo roof (left) which has high IR absorptance at wavelengths across the Planck thermal spectrum for all exit directions compared to an original galvanized lower $E$, lower albedo finish, smoother, much hotter roof (right), which clearly has a large variation in $E(\theta)$ as $\theta$ varies (its higher $T$ is fixed).
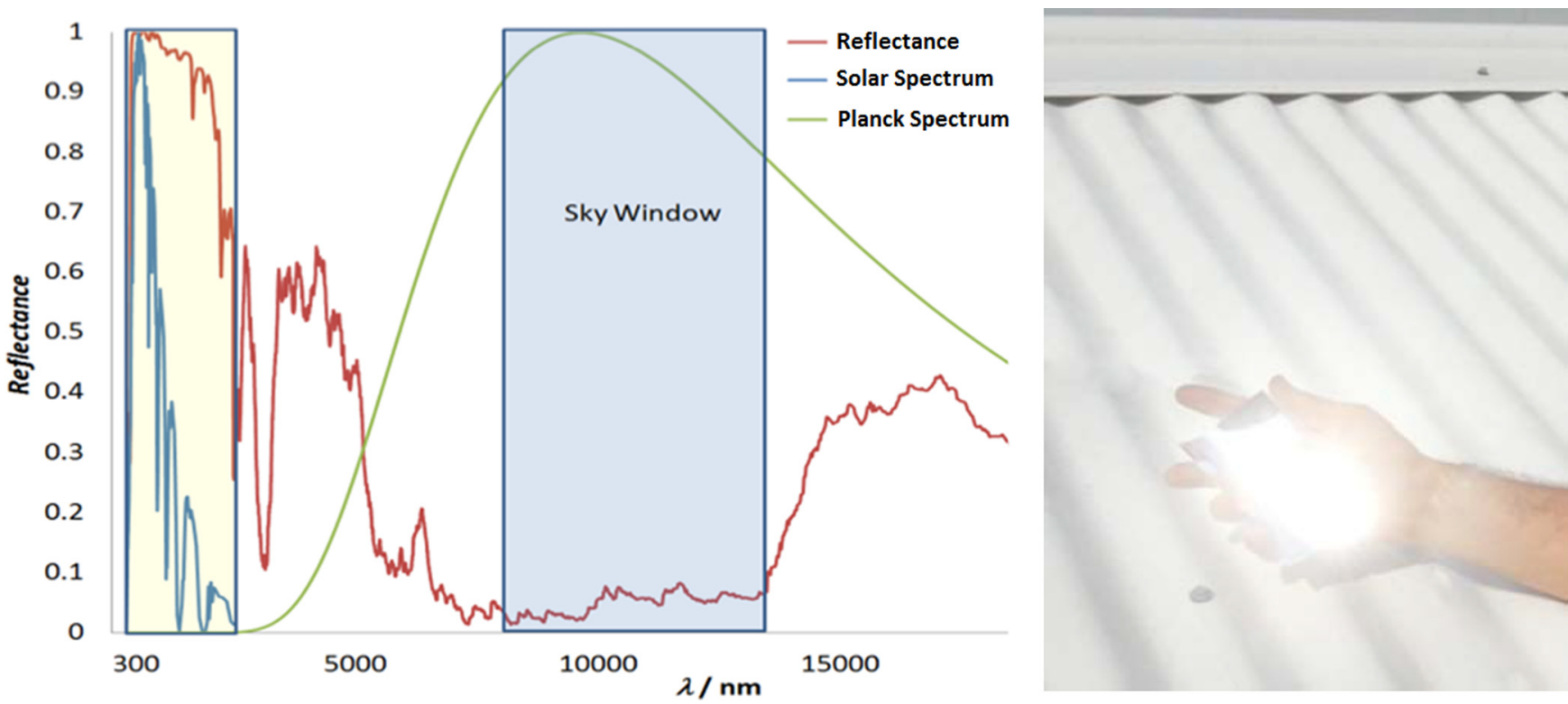

Fig. 3. The reflectance spectrum (red) of our super-cool material across the solar and thermal radiation spectral ranges. Superimposed (scaled to their maximum values) for reference are the AM1.5 solar intensity spectrum (blue) and the Planck black body spectrum (green). $R_{\text {sol }}$ is $97 \%$, and emittance is very high within the clear sky transparent zone, which is shown shaded. The right image shows this surface in summer sunlight above a white commercial cool roof.

radiant heat loss. This needs to be considered in thermal design and in thermal image analysis. A simple experimental test exists which proves the validity of equation (2). It uses a special angular feature in the combination of $E(\theta)$ and calorimetric data which shows that all smooth surface emittances have an almost common angular optical property, namely the representative angle $\theta_{R}$ where $E(\theta, T)=E_{H}[6]$. The solution for smooth $\theta_{R}$ lies universally within $76^{\circ} \pm 3^{\circ}$, whether insulator or conductor. 


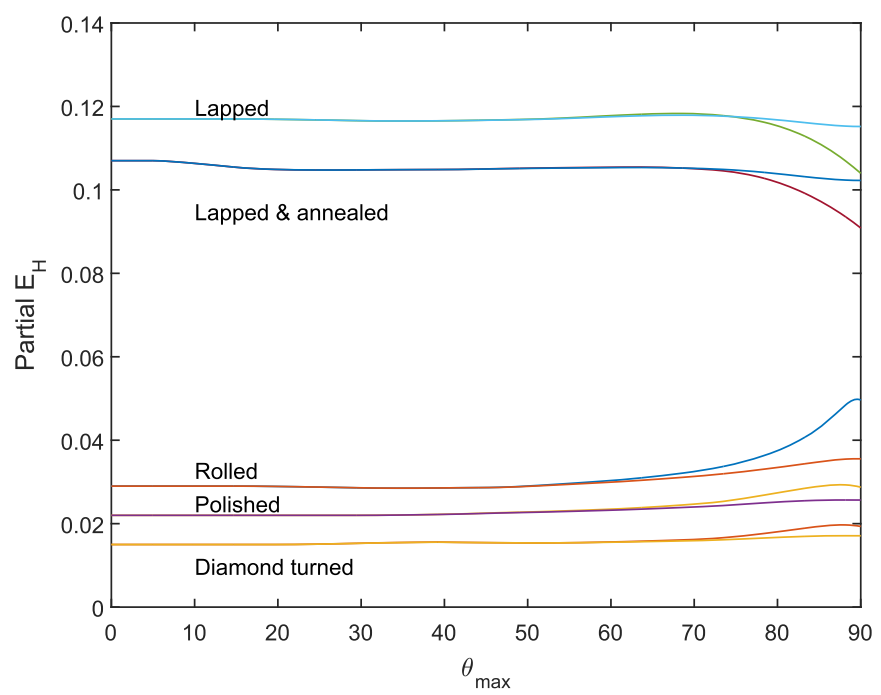

Fig. 4. Emittance of gold with a variety of surface finishes as labeled. The two partial hemispherical plots for each finish are $E_{e}(\Delta \Omega)$ and $E_{p}(\Delta \Omega) . \theta_{\max }=90^{\circ}$ yields $E_{\mathrm{He}}$ and $E_{\mathrm{Hp}} . E_{p}(\Delta \Omega)$ has the much flatter variation with size increase of each hemispherical cap in the high $\theta_{\max }$ range.
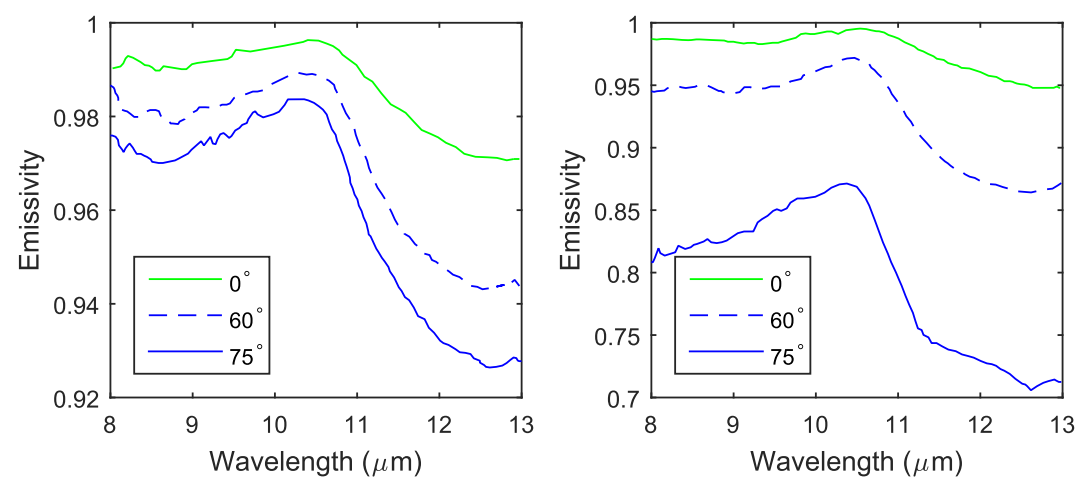

Fig. 5. Large contrast via different vertical axis ranges, between bare smooth ice (right) and course granular snow (left) in angular variations, from normal exit (top curve) to $75^{\circ}$ exit (bottom curve) for spectral emissivity from $8 \mu \mathrm{m}$ to $12.5 \mu \mathrm{m}$. The "free" total emissivity curve lies closer to higher exit angle curves (plots adapted from select data within Fig. 3 of Ref. [8]).

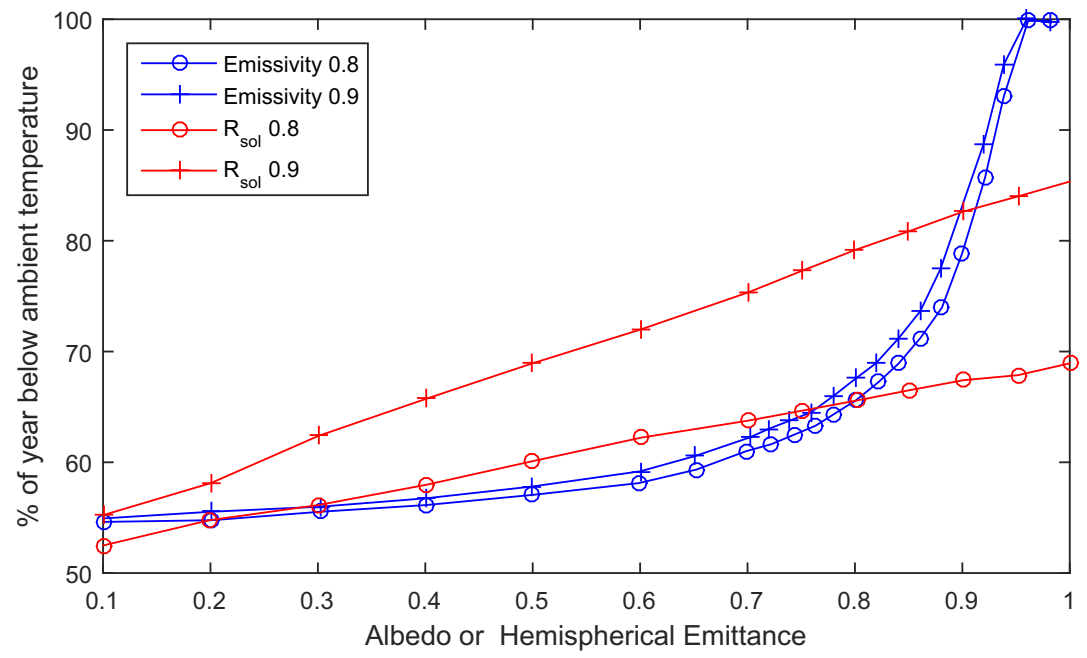

Fig. 6. Plots showing the percentage of time in an average year in Sydney when surfaces will stay below ambient. With albedo of 0.9 and emittance above 0.8 it will do so more than $80 \%$ of the time while with this emittance range and albedo of 0.8 it does so in excess of $65 \%$ of elapsed time. 
Examples follow showing variations for select natural, ceramic and metal surfaces that demonstrate the impact of surface finish on thermal emittance. Metal surface finishes vary according to the wide variety of production and final processing techniques used. Five different processes and surface finishes led to the result in Figure 4 which plots $E_{e}(\Delta \Omega)$ and $E_{p}(\Delta \Omega)$ for gold using data in [7]. $\Delta \Omega$ is confined to hemispherical caps of increasing size determined by the maximum value of the exit angle $\theta$ to the normal. $E_{e}(\Delta \Omega)$ shows the greatest changes for large caps while projected emittance is almost flat. The two roughest surfaces not only have quite different $E(0)$ values but behave in a qualitatively different way as $\Delta \Omega$ approaches $2 \pi$ steradians. Smooth metal always has a significant increase in $E_{e}$ above $E(0)$ but some of their rougher surfaces have total emittance lower than $E(0)$. Total power flows using $E_{p}(2 \pi)$ are around half those using $E_{e}(2 \pi)$. That is, the ratio $P_{p}(2 \pi) / P_{e}(2 \pi)$ varies from 1.0 to lie the range $0.4-0.6$ as $\Delta \Omega$ (cap) increases from 0 to $2 \pi \mathrm{Sr}$ (the exact 0.5 occurs in the $2 \pi$ limit for Lambertian emitters only). Examples of ice and snow angular variations are in Figure 5 [8].

Shiny aluminium as $\mathrm{Al}$ foil, electropolished $\mathrm{Al}$ plate, vacuum-coated $\mathrm{Al}$ on polyester, and polished pure $\mathrm{Al}$ discs have $E_{\mathrm{He}}$ in the range 0.018-0.032 at room temperature [9]. Thus radiant output of $\mathrm{Al}$ on insulator can increase by up to $80 \%$ relative to the best $\mathrm{Al}$. Smooth glass has emittance according to equation (2) and data of 0.75 while we have measured that roughened glass had $E_{H}$ using $E(\theta)$ from an FTIR instrument in the range 0.89-0.91.

\section{Conclusion}

Significant shifts in hemispherical emittance produced by changes in surface finish will lead to a surface staying cooler or hotter for longer. A corollary is that such changes spread over city precincts will have significant impacts on the UHI response. Radiant heat outflow is the main cooling mechanism at night when solar heat gained in the day has to be dissipated fast for human comfort and health. Minimizing solar heat gain in the day, combined with optimum choices of surface and surface finish, can lead to very long periods of sub-ambient or near ambient temperatures as demonstrated in Figure 6 [10].
The authors acknowledge the financial support from the Australian Research Council Discovery grant DP14010200 and SkyCool Pty Ltd, for joint paint studies and Figure 2.

\section{References}

1. A.R. Gentle, G.B. Smith, Is enhanced radiative cooling of solar cell modules worth pursuing? Sol. Energy Mater. Sol. Cells 150, 39 (2016)

2. M. Santamouris et al., Passive and active cooling for the outdoor built environment - analysis and assessment of the cooling potential of mitigation technologies using performance data from 220 large scale projects, Sol. Energy (2016), doi:10.1016/j.solener.2016.12.006

3. T.G. Carter, Issues and solutions to more realistically simulate conventional and cool roofs, in Presented at the Proceedings of Building Simulation: 12th Conference of International Building Performance Simulation Association (IBPSA), Sydney (2011)

4. A.R. Gentle, J.L.C. Aguilar, G.B. Smith, Optimized cool roofs: integrating albedo and thermal emittance with $R$ value, Sol. Energy Mater. Sol. Cells. 95, 3207 (2011)

5. A.R. Gentle, G.B. Smith, A subambient open roof surface under the mid-summer sun, Adv. Sci. 2, 1500119 (2015)

6. A.R. Gentle, G.B. Smith, M.D. Arnold, The representative angle within thermal radiation: a reversibility measure linking radiative cooling rates to surface structure, J. Quant. Spectrosc. Radiat. Transfer (2017), under review

7. W. Sabuga, R. Todtenhaupt, Effect of roughness on the emissivity of the precious metals silver, gold, palladium, platinum, rhodium and iridium, in High Temperatures and Pressures, 15 ECT Proceedings, pp. 861-869 (2001)

8. J. Cheng, Effects of thermal infra-red emissivity directionality on surface broadband emissivity and long wavelength net radiation estimation, IEEE Geosci. Remote Sens. Lett. 11, $499(2014)$

9. M. Donabedian, Emittance of selected materials at cryogenic temperatures, in: Spacecraft Thermal Control Handbook, Vol. II, Cryogenics (The Aerospace Press, California, 2003), chapter 23

10. A.R. Gentle, G.B. Smith, R. Lehmann, C. Crawley, Recent advances in high performance cool roof coatings, in 4th International Conference on Countermeasures to Urban Heat Island (National University of Singapore, Singapore, 2016), to appear in a paper by M.A. Gali et al., focused on super-cool paints, submitted for 2017 SPIE publications

Cite this article as: Geoff B. Smith, Angus R. Gentle, Matthew D. Arnold, Marc A. Gali, Michael B. Cortie, The importance of surface finish to energy performance, Renew. Energy Environ. Sustain. 2, 13 (2017) 